\title{
"Ski"-Shaped Microneedle Facilitates Suturing in a Deep Narrow Surgical Field
}

\author{
Laura Celis ${ }^{1}$, Annelies Mondelaers',3, Andras Csokay, Vincent Van Rompaey5, Tomas Menovsky \\ ${ }^{1}$ Depatment of Ear, Nose and Throat (ENT), St-Vincentius Hospital, Antwerp, Belgium \\ ${ }^{2}$ NeuroVascular Center Antwerp, Antwerp University Hospital, Edegem, Belgium \\ ${ }^{3}$ Department of Translational Neurosciences, University of Antwerp, Antwerp, Belgium \\ ${ }^{4}$ Department of Neurosurgery, Honved Military Hospital, Budapest, Hungary \\ ${ }^{5}$ Department of Ear, Nose and Throat (ENT), Antwerp University Hospital, Antwerp, Belgium \\ ${ }^{6}$ Department of Neurosurgery, Antwerp University Hospital, Edegem, Belgium \\ Email: tomas.menovsky@uza.be
}

How to cite this paper: Celis, L., Mondelaers, A., Csokay, A., Van Rompaey, V. and Menovsky, T. (2020) "Ski"-Shaped Microneedle Facilitates Suturing in a Deep Narrow Surgical Field. Open Journal of Modern Neurosurgery, 10, 364-370. https://doi.org/10.4236/ojmn.2020.103039

Received: June 8, 2020

Accepted: July 10, 2002

Published: July 13, 2002

Copyright $\odot 2020$ by author(s) and Scientific Research Publishing Inc. This work is licensed under the Creative Commons Attribution International License (CC BY 4.0).

http://creativecommons.org/licenses/by/4.0/

(c) (i) Open Access

\begin{abstract}
Background: Microsurgical suturing requires high level of dexterity and practice. Suturing in a deep and narrow operative field poses additional difficulties mainly due to the long microsurgical instruments and the difficulty of manipulating a curved needle. Objective: In this technical note, we describe a simple modification of a classical curved suture needle into a "ski"-shaped needle. Methods: A classical curved needle is stretched using a needle holder. Only the tip of the needle is left slightly bent, imitating a ski. Once the needle is bent into the ski shape, it can be grasped virtually in any position with the needle holder. Results: We have used the ski needle technique suturing in several patients in which the operative field was deep and limited such as dura repair in spinal surgery in obese patients, facial nerve grafting in the internal acoustic meatus and posterior inferior cerebellar artery suturing between the caudal cranial nerves. Conclusion: This modification facilitates free positioning of the needle in the needle holder and as a consequence results in easy and faster microsuturing in a deep and narrow operative field. The ski needle technique was felt to be superior to the classical curved needle technique.
\end{abstract}

\section{Keywords}

Microneedle, Facial Nerve Grafting, Microsurgery, Deep Surgical Field, Ski

\section{Introduction}

Microsurgical suturing requires high level of dexterity and practice. Suturing in a 
deep and narrow operative field poses additional difficulties mainly due to the long microsurgical instruments and the difficulty of manipulating a curved needle. For optimal suturing with a traditional curved needle, the needle is held in a needle holder in a specific position that is perpendicular to the needle holder axis (Figure 1).

Suturing with this "classical" position of the needle requires certain degree of space and angle for the instruments. For a superficial surgical field this is no issue. In a deep narrow field such as in neurosurgery and/or Ear, Nose and Throat (ENT), there is a significant limitation of movements and angulations of the instruments due to the restricted operating field and long bayonet instruments (Figure 2).

In such situations, a curved suture needle cannot be moved and twisted in unlimited directions as one would wish. The more the needle holder is coming from a perpendicular direction into a narrow surgical field, the more it becomes demanding to suture. In general, only three grips are possible with the curved needle. The first and second one is a $90^{\circ}$ position (forehand and backhand) and the third one is parallel with the needle holder but only at the end of the needle (Figures 3(A)-(C)).

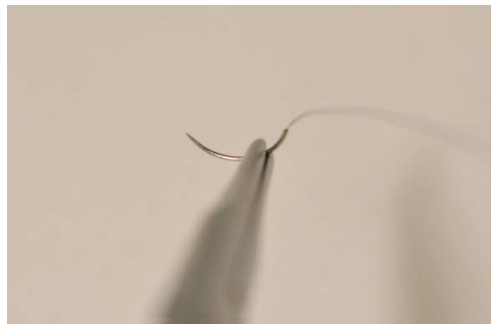

Figure 1. Curved needle correctly positioned in a needle holder.

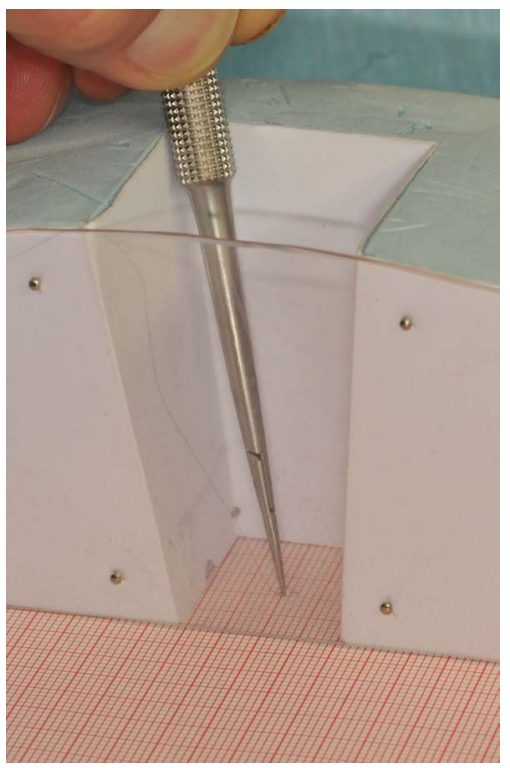

Figure 2. In a deep narrow field the position of the needle within the needle holder is depending on the position of the needle holder itself. 


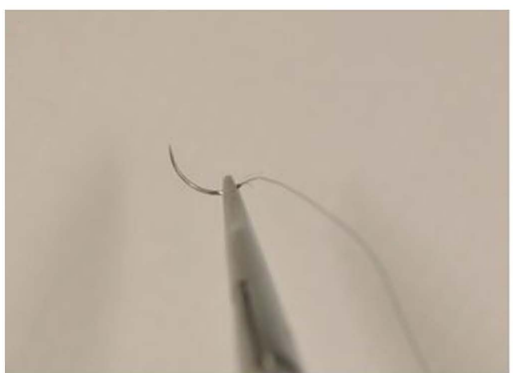

(A)

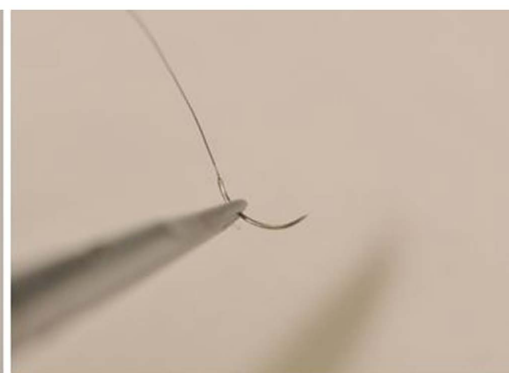

(B)

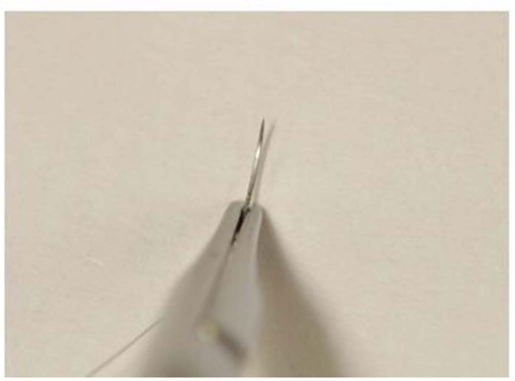

(C)

Figure 3. Classical curved needle can be held only in more or less three positions in the needle holder, i.e. (A) $90^{\circ}$ forehand; (B) $90^{\circ}$ backhand; and (C) parallel with the needle holder, but only at the end of the needle. The last grip and position is very unpractical for suturing.

The last grip and position although is very unpractical for suturing. This limitation in free positioning of the needle strongly limits its insertion and exertion of the needle through the tissue. Another position of a curved needle besides the three mentioned grips in the needle holder is not easy as the curvature of the needle more or less determines its grip and position in the needle holder. As a consequence, holding the needle over or under $90^{\circ}$ is not possible and the needle will flip back into a $90^{\circ}$ position.

In this technical note, we describe a simple modification of a classical curved suture needle into a "ski"-shaped needle. This modification facilitates free positioning of the needle in the needle holder and makes microsuturing in a deep and narrow operative field feasible and easier.

\section{Materials and Methods}

The modification of a standard curved microneedle into a "ski"-shaped needle is performed under the operating microscope using a middle-sized needle holder. The curved needle is stretched using the needle holder as depicted in Figure 4.

Only the tip of the needle is left slightly bent, imitating a ski. Once the needle is bent into the ski shape, it can be grasped virtually in any position with the needle holder.

The direction of the ski-needle in the needle holder can be almost $360^{\circ}$ in the $\mathrm{x}$ plane and $360^{\circ}$ in the $\mathrm{z}$-plane depending on the rotation of the needle holder (Figure 5). The ski needle requires only pushing motion to advance it through tissue and no rotating movements. 


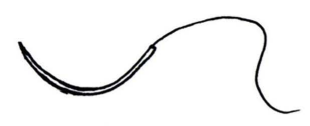

(A)

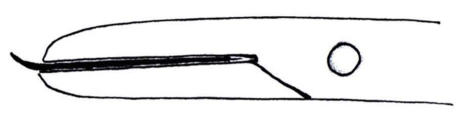

(C)

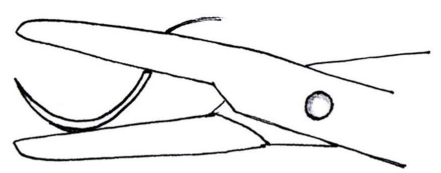

(B)

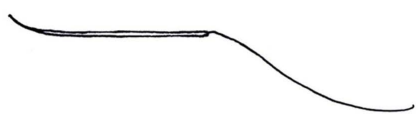

(D)

Figure 4. The curved needle is stretched using a middle-sized needle holder. Only the tip of the needle is left slightly bent, imitating a ski.

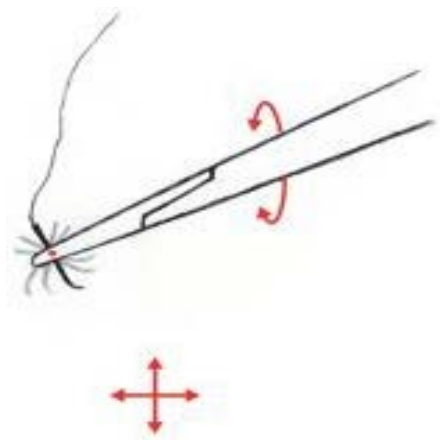

Figure 5. The direction of the "ski"-shaped needle in the needle holder can be almost $360^{\circ}$ in the $\mathrm{x}$ plane and $360^{\circ}$ in the z-plane depending on the rotation of the needle holder. The ski needle requires only pushing motion to advance it through tissue and no rotating movements.

\section{Results}

We have used the ski needle technique suturing in several patients in which the operative field was deep and limited such as dura repair in spinal surgery in obese patients, facial nerve grafting in the internal acoustic meatus (Figure 6) and posterior inferior cerebellar artery suturing between the caudal cranial nerves. The ski needle technique was felt to be superior to the classical curved needle technique.

\section{Discussion}

Performing microsurgical suturing requires high level of dexterity and practice. In neurosurgery and ENT, microsuturing in deep and narrow operative field poses additional difficulties mainly due to 1 ) limited space confined by important structures such as cranial nerves, blood vessels, and brain tissue, 2) the required long bayonet microsurgical instruments, 3) the physiological tremor of 


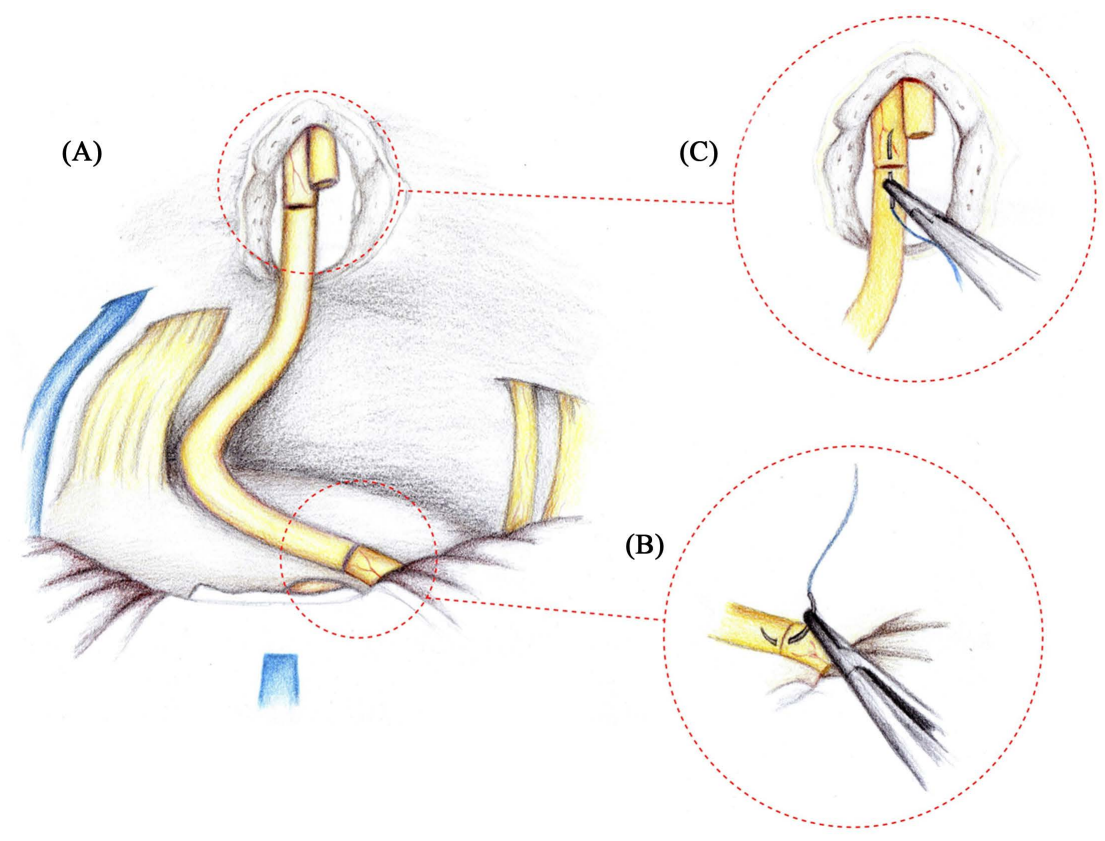

Figure 6. (A) Schematic drawing of suturing a facial nerve graft in the internal acoustic meatus in a 25 -year-old man after resection of a large vestibular schwannoma in which the facial nerve was not discernible. The facial nerve as reconstructed using a sural nerve graft; (B) The proximal part could be sutured with a classical curved needle correctly positioned in a needle holder due to the optimal position of the suture line; (C) The distal nerve end was sutured deep in the internal acoustic meatus. With a classical needle in a needle holder, the direction of the needle was unsuitable for suturing, regardless how the needle holder was placed or twisted. The distal suture line was performed with a ski needle using a push and pull technique.

the surgeon that is amplified due to long shafts of the instruments, and 4) the difficulty of manipulating a curved needle.

The latter is due to the limitation of movements and angulations of the instruments and the limited position of the curved needle in the needle holder. Consequently, a curve needle cannot be moved and twisted as one would wish in the depth. To pass a curved needle through tissue is only possible with rotating movement of the needle holder. If the direction of the needle towards the sutured tissue is not optimal, and that is hardly the case, such rotating movement has no sense and results in an undesirable or even impossible angle of entry into the tissue. In other words, a curve needle held in a classical needle holder limits the appropriate insertion of the needle into the tissue that needs to be sutured. Time and effort are lost by re-positioning and grasping the needle and thus abnormal and unnecessary movements are performed.

Other than the classic curved needles are not common or available in microsurgery. Ski or so-called "take-off" needle exists mainly in laparoscopic or endoscopic surgery when passing the needle through an endoscope is mandatory [1]. This needle is only commercially available in macro format. A solution for microsurgery is to alter a commercially available curved needle into a ski-shaped needle [2]. This modification can be easy and fast performed by any micro- 
surgeon.

The advantages of using a "ski"-shaped needle for microsurgery in deep operative field are several. First of all, ski needle in a deep field gives the surgeon more movement and insertion freedom. The ski needle can be pushed or pulled in almost any direction independent of the position in the needle holder and does not to be rolled or twisted, which is actually impossible when suturing in a deep narrow field with long bayonet instruments. This is not possible with the curved needle as shown in Figure 2. In addition, in some circumstances, the ski needle is easier to introduce into a narrow surgical field than a curved needle.

Second advantage of a ski needle is that it is easy to grasp and that it will not rotate in the needle holder. Grip is always good regardless of its position in the needle holder. The ski needle results in more precise placement of the sutures.

Finally, using a ski needle saves some time. Time is not lost by re-positioning and grasping the needle and abnormal movements such as with a curved needle. The average time of suturing with the ski needle seems shorter, although we did not quantify this.

We have used the ski needle in several instances in which the operative feel was deep and space limited such as in repair in spinal surgery in obese patients. Most of such leaks in spinal surgery occur laterally of the nerve root which means at the deepest point and the space is confined by the medial edge of the pedicle and the dural sac medially. The bony edges of the vertebra prevent adequate exposure and the ski needle is definitely of use. Other indications include microvascular suturing (e.g. bypass operation such as superficial temporal artery (STA)-P2 bypass or Posterior Inferior Cerebellar Artery (PICA) bypass) and nerve suturing in deep cisternal spaces such as facial nerve grafting in the internal acoustic meatus as illustrated in Figure 6.

\section{Conclusion}

In this technical note, we describe a simple modification of a classical curved suture needle into a "ski"-shaped needle. This modification facilitates free positioning of the needle in the needle holder and makes microsuturing in a deep and narrow operative field feasible and easier.

\section{Acknowledgements}

The authors would like to thank Miss Lemieh Hajjab for the drawings and Andy Van Rompaey for the photographs.

\section{Conflicts of Interest}

The authors declare no conflicts of interest regarding the publication of this paper.

\section{References}

[1] Tagaya, N., Mikami, H., Nakano, S., Shimoda, M. and Kogure, H. (2000) The Use of 
Double-Straight Needle Device in Laparoscopic Incisional and Ventral Hernia Repair. Surgical Laparoscopy Endoscopy \& Percutaneous Techniques, 10, 187-189. https://doi.org/10.1097/00019509-200006000-00018

[2] Csokay, A., Imreh, D., Papp, A. and Valalik, I. (2012) Straight Needle with Fingertip Support Technique Reduces Exclusion Time during Bypass (In Vitro, In Vivo Animal Study): A Technical Note. Acta Neurochirurgica (Wien), 154, 1851-1854.

https://doi.org/10.1007/s00701-012-1468-0 